\title{
Characterization of Endophytic Pseudomonas Sp. 16 Strain and Its Use for the Enhancement of Phytoextraction
}

\author{
Tomasz Płociniczak, Iwona Mikołajczyk, Zofia Piotrowska-Seget \\ University of Silesia, Department of Microbiology \\ Jagiellonska 28, 40-032 Katowice, Poland \\ tomasz.plociniczak@us.edu.pl; mikolajczyk.iwona@o2.pl; zofia.piotrowska-seget@us.edu.pl
}

\section{Extended Abstract}

Environmental pollution by heavy metals has become a serious problem in the world. Phytoextraction, which is one of the plant-based technologies has attracted the most attention for the bioremediation of soils polluted with these contaminants. The success of metal extraction depends on many factors, but the most important are a plant's ability to uptake and translocate metals to its stems and leaves, metal bioavailability and soil type. The results of many studies have shown that the efficiency of heavy metal phytoextraction may be supported by metal-resistant bacteria that belong to the plant growth-promoting bacteria (PGPB) group. Among them plant growth-promoting endophytes (PGPE) may confer plants with a higher tolerance to heavy metal stress and may stimulate the growth of the host plant through several mechanisms.

The aim of this study was to estimate the potential of a metal-resistant strain of Pseudomonas sp. 16, isolated from Silene vulgaris tissues, to enhance $\mathrm{Zn}, \mathrm{Cd}$ and $\mathrm{Cu}$ uptake by white mustard under laboratory conditions. Moreover, its potential to promote the growth of plants was also determined. Additionally, the ability of spontaneous rifampicin-resistant mutant of tested strain to colonize the internal tissues of Sinapis alba and its survival after introduction into soil were also determined.

The strain showed the high resistance to $\mathrm{Cd}, \mathrm{Zn}$ and $\mathrm{Cu}$ and produced large and clear orange haloes around the colonies grown on CAS agar, which indicates siderophore production. The activity of ACCD was $2.3 \mu$ mol $\alpha$-ketobutyrate $\mathrm{mg}^{-1} \mathrm{~h}^{-1}$. The strain also produced IAA at a concentration of $6.96 \pm 2.41 \mu \mathrm{g}$ IAA mL ${ }^{-1}$ and was able to solubilize $\mathrm{Ca}_{3}\left(\mathrm{PO}_{4}\right)_{2}$ in an NBRIP medium. The halo size was $13.67 \pm 1.15 \mathrm{~mm}$. The presence of the color orange on the paper filter placed on agar plates supplemented with glycine demonstrated the ability of the tested strain to release hydrocyanic acid. Moreover, this strain exhibited extracellular cellulase activity and was capable of motility and these features facilitated the plant colonization by bacteria. Plant growth-promoting features of tested strain caused higher biomass and heavy metal accumulation by white mustard growing in soil inoculated with suspension of tested strain, as compared with control plants growing in soil treated with suspension of thermal inactivated strain.

A metal-tolerant Pseudomonas sp. 16 strain, due to the activity of several mechanisms that are considered to be important for plant growth-promotion has the potential to enhance the phytoextraction of heavy metals from contaminated soil. Additionally, tested strain showed a high survival rate after introduction into the soil and was able to colonize the internal tissues of $S$. alba. These features indicate that Pseudomonas sp. 16 strain in combination with $S$. alba can be regarded as an effective tool for the phytoextraction of heavy metals.

The research was supported by grant No. 2013/11/B/NZ9/00152 financed by National Science Centre (Poland). 